\title{
Cavernosal Abscess due to Streptococcus Anginosus: A Case Report and Comprehensive Review of the Literature
}

\author{
Caitlin M. Dugdale ${ }^{a} \quad$ Andrew J. Tompkins ${ }^{b} \quad$ Rebecca M. Reece ${ }^{c} \quad$ Adrian F. Gardner \\ Department of alnternal Medicine, bUrology and Infectious Disease, Brown University, Providence, R.I., USA
}

\section{Key Words \\ Corpus cavernosum • Penile abscess • Cavernosal abscess • Necrotizing cavernositis $\cdot$ Streptococcus anginosus}

\begin{abstract}
Corpus cavernosum abscesses are uncommon with only 23 prior reports in the literature. Several precipitating factors for cavernosal infections have been described including injection therapy for erectile dysfunction, trauma, and priapism. Common causal organisms include Staphylococcus aureus, Streptococci, and Bacteroides. We report a unique case of a corpus cavernosum abscess due to proctitis with hematological seeding and review the literature on cavernosal abscesses.

Copyright $\odot 2013$ S. Karger AG, Basel
\end{abstract}

\section{Introduction}

Abscesses of the corpus cavernosum are uncommon infections that usually present with several days of progressive penile pain and swelling. While many corpus cavernosum abscesses have no identifiable trigger, cases have been reported in association with intracavernous injection therapy [1-5], foreign bodies [6], perianal [7] or perineal abscess drainage $[8,9]$, intra-abdominal abscess

\section{KARGER}

Fax +4161306 1234

E-Mail karger@karger.ch

www.karger.com
(C) 2013 S. Karger AG, Base

1015-9770/13/0071-0051\$26.00/0

Accessible online at:

www.karger.com/cur extension [10], priapism [2, 11], and even hematological seeding from periodontal abscesses [12-14]. Diabetic patients are at greater risk for penile abscesses, likely due to microvascular disease and relative immune system suppression. Typical causative organisms include Staphylococcus aureus, Streptococci, Bacteroides, and Enterococci, though cases involving Mycobacterium tuberculosis, Escherichia coli, Klebsiella, Actinomyces, and other anaerobes have been described. Most cavernosal abscesses are treated with incision and drainage in addition to systemic antibiotics, though several authors have reported successful treatment with aspiration alone [1, $8,15]$. We report a case of corpus cavernosum abscess caused by Streptococcus anginosus in the setting of proctitis, with surgical treatment complicated by abscess recurrence and cutaneous fistula formation.

\section{Case Report}

A 48-year-old previously healthy man presented to the emergency department with a 3-week history of right groin pain and a 1-week history of fevers, chills and night sweats. Abdominal and pelvic CT scan with intravenous contrast showed perirectal stranding suggestive of proctitis, so he was discharged on oral ciprofloxacin and metronidazole. Three days later, he noted penile swelling and a fever to $40.4^{\circ} \mathrm{C}$ and he returned to the emergency department. He denied any abdominal pain, hematuria, genital lesions, or difficulty urinating. The patient reported a history of untreated erectile dysfunction for the past year, but denied new sexual partners, genital trauma, or past sexually transmitted infections. 
Table 1. Abscess aspirate culture data

\begin{tabular}{ll}
\hline Initial presentation & Recurrence (3 months later) \\
\hline 4+ Streptococcus anginosus (SMG) & 2+ Streptococcus constellatus (SMG) \\
1+ Yeast & 1+ Methicillin resistant Staphylococcus aureus \\
1+ Gram negative rods & 2+ Beta-hemolytic Streptococcus, Group B \\
1+ Coagulase-negative Staphylococcus & 2+ Anaerobic gram negative rods \\
2+ Mixed anaerobes & \\
\hline
\end{tabular}

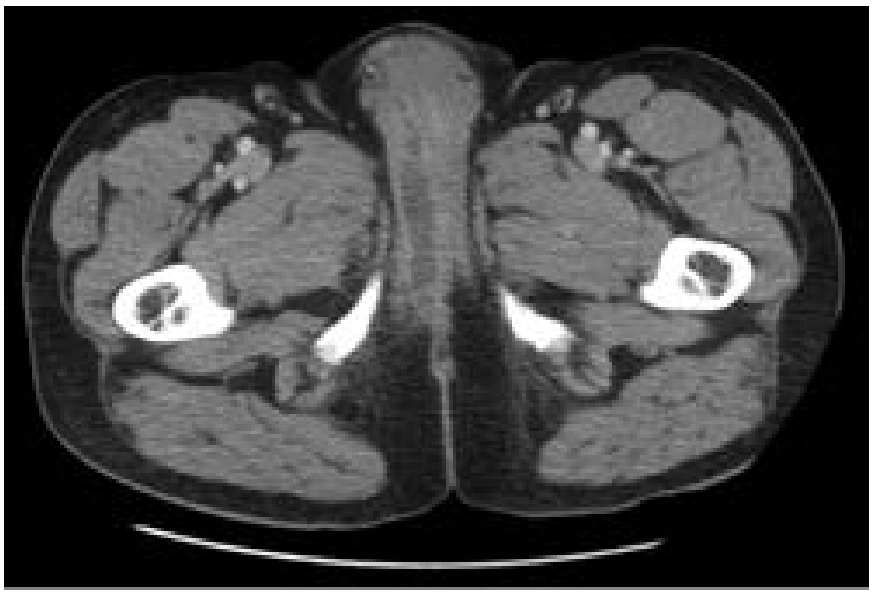

Fig. 1. Axial CT shows a right corpus cavernosum abscess.

Upon arrival to the emergency department, he was afebrile, but tachycardic. Digital rectal exam demonstrated an enlarged, non-tender prostate. Mild penile swelling at the right base was observed with induration and tenderness along the right penile shaft. There was no overlying erythema or ulceration and testicular exam was normal. Laboratory tests were notable for leukocytosis $(26.3 \times 10 \% \mathrm{ml})$ with $8 \%$ bands and a mild normocytic anemia. Chemistries including lactate, lipase and urinalysis were unremarkable. Rapid HIV testing was non-reactive.

Repeat CT scan of the abdomen and pelvis showed multiple new splenic infarcts and a fluid collection within the right corpus cavernosum with punctate pockets of gas suggestive of an abscess (fig. 1). The presence of splenic infarcts raised concern for an intravascular source of infection (e.g. endocarditis) with potential embolic seeding of the right corporal body. However, transesophageal echo was negative for vegetations. Empiric therapy with intravenous meropenem and clindamycin was initiated.

Bedside aspiration of the right corporal body returned $10 \mathrm{ml}$ of purulent fluid that grew Streptococcus anginosus, yeast, coagulase negative Staphylococcus, and mixed anaerobes (table 1). Urine cultures yielded no growth, but blood cultures grew mi- croaerophilic Streptococcus. The patient underwent incision and drainage of the corpus cavernosum abscess under general anesthesia with the placement of a Penrose drain. When aspirate sensitivities returned, his therapy was narrowed to intravenous ceftriaxone and oral metronidazole. The Penrose drain was removed after 5 days and he completed a 6-week course intravenous ceftriaxone and oral metronidazole as an outpatient out of concern for an intravascular source of infection.

Two months later, the patient developed a cutaneous draining fistula tract in the area of the prior drain. He was taken back to the operating room for perineal and right corporal body exploration with closure of the fistula tract. However, his postoperative course was complicated by worsening pain, redness, and swelling around the operative site. Pelvic CT scan demonstrated a recurrent abscess in the right corpus cavernosum. Repeat drainage was performed and aspirate cultures grew several organisms, including Streptococcus constellatus (table 1). He was discharged on ampicillin-sulbactam and trimethoprim-sulfamethoxazole for an additional 3 weeks. Although the recurrent abscess and fistula tract resolved, the patient did report erectile dysfunction and right testicular numbness at follow-up.

\section{Discussion}

While many abscesses of the corpus cavernosum are idiopathic [15-21], cases have been described in association with priapism $[2,11]$, alprostadil or papaverine injections [1-5], trauma [9], tuberculosis [22, 23], penile prosthesis placement [6], and intra-abdominal abscesses [10]. There have also been case reports of cavernosal abscesses following perineal [8, 9] and perianal [7] abscess drainage, presumably via extension through Buck's fascia. Although the corpora cavernosa are not typical sites of hematological spread of infection, Pearle et al. [12] reported a case of cavernosal abscess secondary to a dental abscess with $S$. anginosus bacteremia. Similarly, Charles et al. [14] described an abscess of the corpus cavernosum due to either fellatio or a periodontal abscess with associated $S$. constellatus bacteremia. Sater et al. [13] also reported a case of cavernosal abscess with dental caries as the presumed source. 


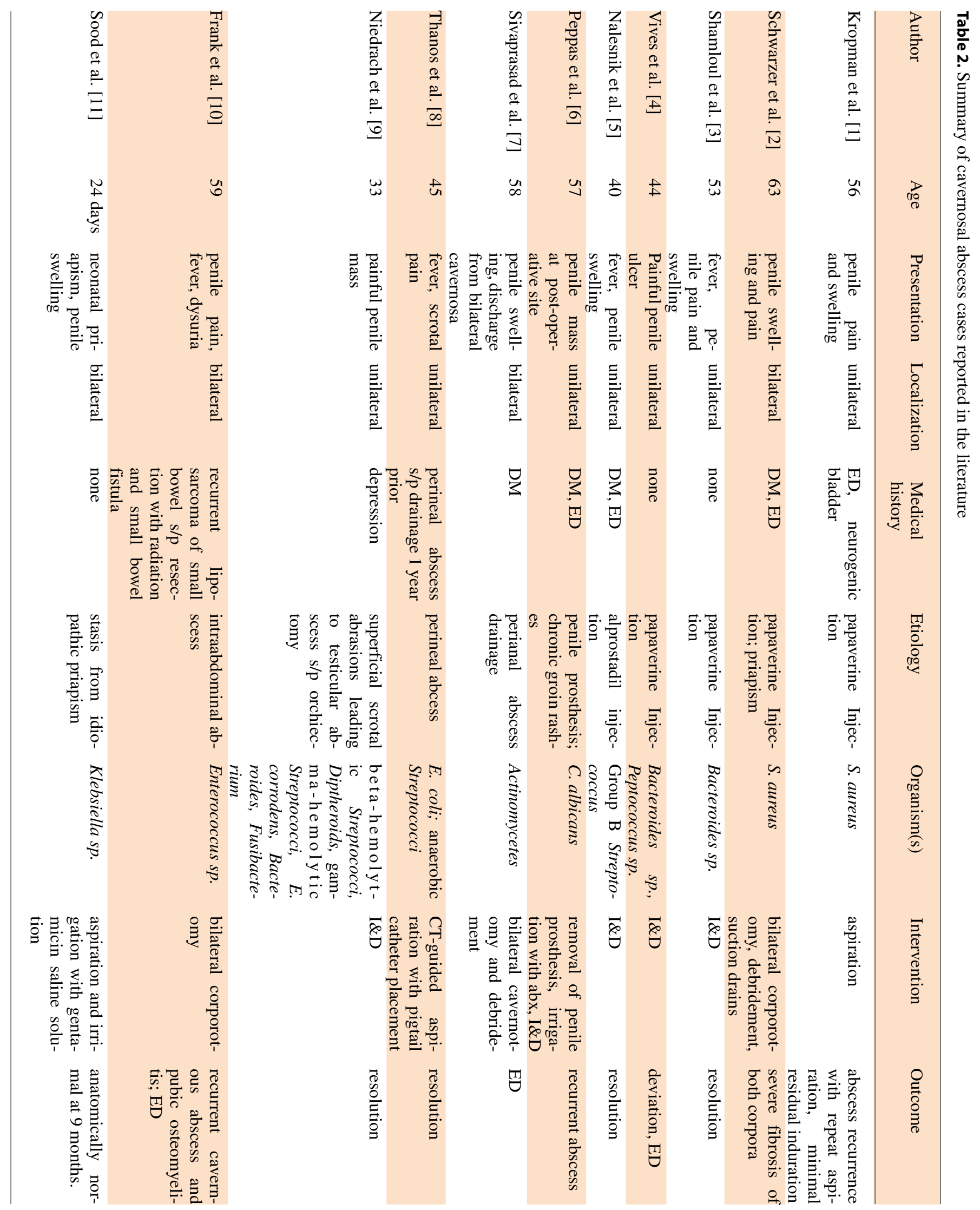




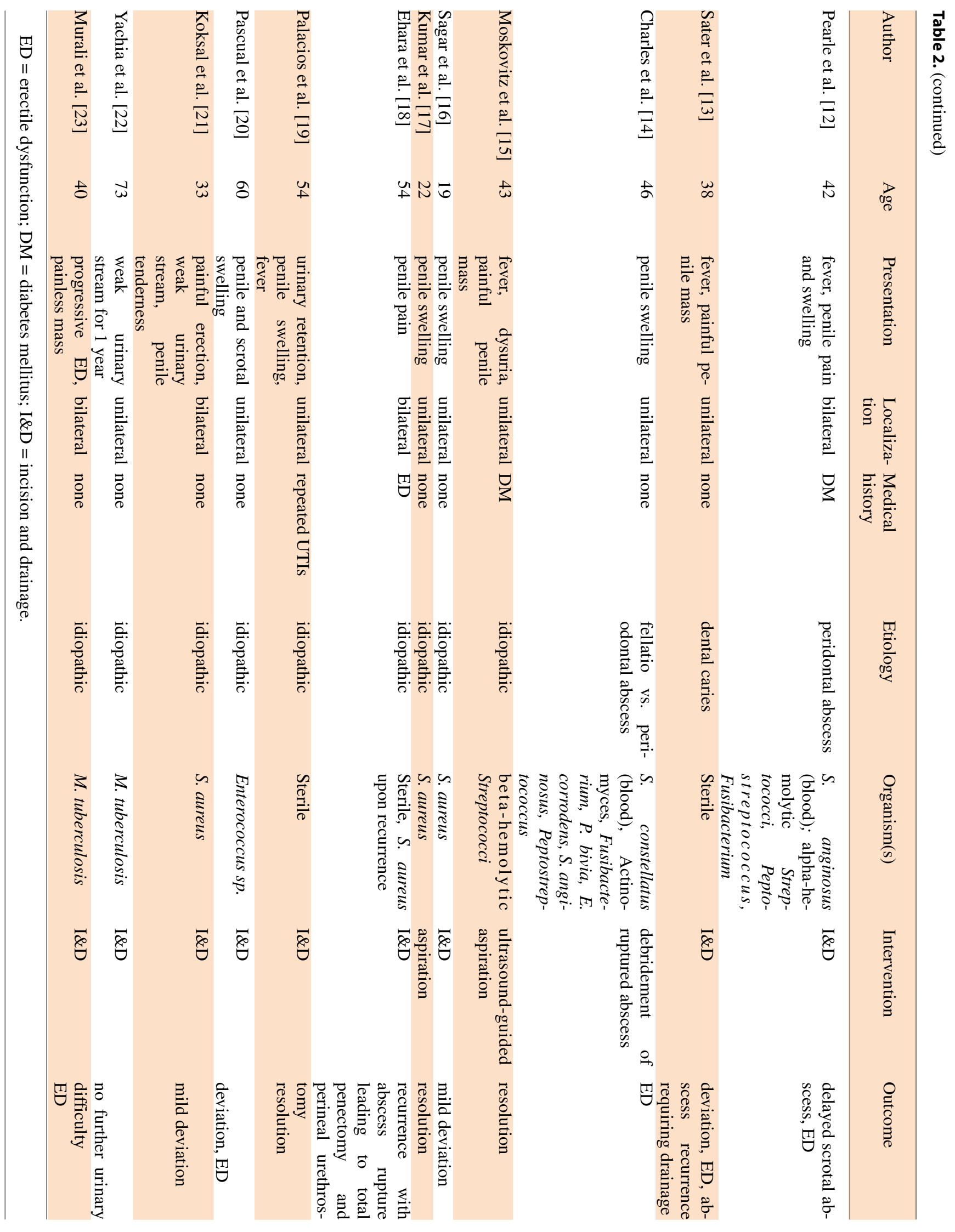


The Streptococcus milleri group (SMG) bacteria, including $S$. anginosus, $S$. constellatus, and $S$. intermedius, are known for their ability to cause deep tissue abscesses $[24,25]$. Systemic SMG infections are often associated with breakdown in gastrointestinal epithelium, as in dental abscesses [26], ingested foreign bodies [24], and gastrointestinal malignancies [27-29]. Murarka et al. [30] reported a case of liver abscess secondary to disseminated $S$. anginosus from sigmoid diverticulitis. Our patient had evidence of proctitis on CT scan, which was the likely source of $S$. anginosus bacteremia with possible intravascular infection subsequently seeding the corpus cavernosum.

A review of the literature revealed 23 reported cases of cavernosal abscess (table 2). The patients' mean age was 45 years (range 19-73 years). Penile pain and swelling were the most common presenting symptoms and one-third of abscesses were bilateral. Although over one-third of cases were spontaneous, other etiologies included intracavernous injection (22\%), associated perianal/perineal/intra-abdominal abscess (13\%), and dental infections with hematologic spread (13\%). There were also individual cases attributable to a penile prosthesis, priapism, and genital trauma. The most common causal organisms were $S$. aureus (25\%), Streptococci (21\%), Fusibacteria (13\%) and Bacteroides (13\%). Diabetic patients accounted for $25 \%$ of reported cases. However, half of diabetic patients also used intracavernous injection therapy for erectile dysfunction, further increasing their risk for abscess formation.

Corpus cavernosum abscesses are generally treated with incision and drainage followed by broad-spectrum antibiotics. While most patients regain normal anatomical and erectile function following abscess drainage, many do experience penile deviation [16, 20, 21], erectile dysfunction [7, 10,12,14, 20], or abscess recurrence $[6,10,13,18]$. Shamloul et al. [3] reported a case of cavernositis in which the patient presented within 36 hours of symptom onset, and abscess drainage resulted in no loss of erectile function. This case suggests that early diagnosis and treatment of cavernosal abscess may improve the likelihood of preserved erectile function, as there is less cavernosal necrosis and fibrosis prior to surgical intervention.

Although traditional therapy for cavernosal abscesses has focused on surgical drainage, less invasive interventional techniques may offer a lower risk for longterm sequelae. Thanos et al. [8] described a case of a cavernosal abscess that was successfully treated with CT-guided aspiration and pigtail catheter placement as well as broad-spectrum antibiotics. The procedure was performed under local anesthesia with minimal trauma to the corpus cavernosum. They reported complete resolution of the abscess with no resultant erectile dysfunction. Kropman et al. [1] and Moskovitz et al. [15] also reported abscess resolution with aspiration followed by systemic antibiotics. This conservative approach is particularly appealing in light of the risk of erectile dysfunction, penile deviation, and fibrosis of the corpus cavernosum with aggressive surgical intervention. However, given the risk of cavernosal fibrosis and abscess recurrence with incomplete evacuation of the abscess, incision and drainage remains the mainstay of therapy.

\section{Conclusion}

Abscess of the corpus cavernosum is an uncommon infection that is frequently idiopathic, but may also be a result of intracavernosal injection, perineal abscess extension, and septic metastases. It should be considered in the differential for acute onset of penile pain and swelling, particularly in diabetic patients. Prompt diagnosis and treatment may reduce the risk of long-term sequelae that result from cavernosal fibrosis. Surgical drainage is the most widely accepted treatment, but carries a substantial risk of erectile dysfunction and penile deviation. 


\section{References}

$\checkmark 1$ Kropman RF, de la Fuente RB, Venema PL, van Imhoff WL: Treatment of corpus cavernosum abscess by aspiration and intravenous antibiotics. J Urol 1993;150:1502-1503.

-2 Schwarzer JU, Hofmann R: Purulent corporeal cavernositis secondary to papaverine-induced priapism. J Urol 1991;146:845-846.

-3 Shamloul R, Kamel I: Early treatment of cavernositis resulted in erectile function preservation. J Sex Med 2006;3:320-322.

$\checkmark 4$ Vives A, Collado A, Ribe N, Segarra J, Ruiz Castane E, Pomerol JM: Cavernositis following intracavernous injection of vasoactive drugs. Urol Int 2001;67:111-112.

$>5$ Nalesnik JG, Jones L, Kraus SR: Group B streptococcal septicemia following intracavernous injection therapy for erectile dysfunction in diabetes. J Urol 2004;172:151-152.

6 Peppas DS, Moul JW, McLeod DG: Candida albicans corpora abscess following penile prosthesis placement. J Urol 1988;140:1541 1542.

7 Sivaprasad G, Devanathan KS, Ganesh G: Corpora cavernositis caused by actinomycetes. Scand J Urol Nephrol 2005;39:93-94.

8 Thanos L, Tsagouli P, Eukarpidis T, Mpouhra $\mathrm{K}$, Kelekis D: Computed tomography-guided drainage of a corpus cavernosum abscess: a minimally invasive successful treatment. Cardiovasc Intervent Radiol 2011;34:217-219.

$>9$ Niedrach WL, Lerner RM, Linke CA: Penile abscess involving the corpus cavernosum: a case report. J Urol 1989;141:374-375.

10 Frank I, Lieber MM: Gas containing cavernous abscess secondary to an intra-abdominal abscess. J Urol 1999;162:1382-1383.

$>11$ Sood R, Wadhwa SN, Jain V: Neonatal priapism associated with spontaneous bilateral pyocavernositis. Ann Acad Med Singapore 2006;35:425-427.
12 Pearle MS, Wendel EF: Necrotizing cavernositis secondary to periodontal abscess. J Urol 1993;149:1137-1138.

13 Sater AA, Vandendris M: Abscess of corpus cavernosum. J Urol 1989;141:949.

14 Charles T, Roy C, Lang H, Jacqmin D, Hansmann Y: Abscess of corpus cavernosum following a fellatio or a periodontal infection. Curr Urol 2009;3:217-219.

-15 Moskovitz B, Vardi Y, Pery M, Bolkier M, Levin DR: Abscess of corpus cavernosum. Urol Int 1992;48:439-440.

16 Sagar J, Sagar B, Shah DK: Spontaneous penile (cavernosal) abscess: case report with discussion of aetiology, diagnosis, and management with review of literature. ScientificWorldJournal 2005;5:39-41.

17 Kumar S, Sharma SK, Goswami AK: Spontaneous bacterial abscess of corpus cavernosum. Indian J Urol 2004;20:66-67.

18 Ehara H, Kojima K, Hagiwara N, Phuoc NB, Deguchi T: Abscess of the corpus cavernosum. Int J Infect Dis 2007;11:553-554.

19 Palacios A, Masso P, Versos R, Osorio L, Carvalho LF, Soares J, Marcelo F: penile abscess. Case report. Arch Esp Urol 2006;59: 809-811.

20 Pascual Regueiro D, Garcia de Jalon Martinez A, Mallen Mateo E, Sancho Serrano C, Borque Fernando A, Rioja Sanz LA: penile curvature secondary to cavernous body abscess. Actas Urol Esp 2003;27:55-59.

-21 Koksal T, Kadioglu A, Tefekli A, Usta M, Besisik A, Erol B: Spontaneous bacterial abscess of bilateral cavernosal bodies. BJU Int 1999;84:1107-1108.

22 Yachia D, Friedman M, Auslaender L: Tuberculous cold abscess of the corpus cavernosum: a case report. J Urol 1990;144:351-352.
23 Murali TR, Raja NS: Cavernosal cold abscess: a rare cause of impotence. Br J Urol 1998:82:929-930.

24 Nisbet M, Thomas M: Liver abscess associated with persistent Streptococcus anginosus bacteremia. Clin Infect Dis 2005;41:352-353, 403-405.

25 Bert F, Bariou-Lancelin M, Lambert-Zechovsky N: Clinical significance of bacteremia involving the "Streptococcus milleri" group: 51 cases and review. Clin Infect Dis 1998;27: 385-387.

26 Karchmer AW, MacGillivray TE, Healey TT, Stone JR: Case records of the massachusetts general hospital. Case 1-2011. A 35-year-old man with fever, bacteremia, and a mass in the left atrium. N Engl J Med 2011;364:158-166.

27 Rich MW, Radwany SM: 'Streptococcus milleri' septicemia in a patient with colorectal carcinoma. Eur J Clin Microbiol Infect Dis 1993; $12: 225$

28 Sasaki H, Ishizuka T, Muto M, Nezu M, Nakanishi Y, Inagaki Y, Watanabe $\mathrm{H}$, Watanabe H, Terada M: Presence of Streptococcus anginosus DNA in esophageal cancer, dysplasia of esophagus, and gastric cancer. Cancer Res 1998;58:2991-2995.

29 Muto M, Ohtsu A, Boku N, Tajiri H, Yoshida $\mathrm{S}$ : Streptococcus milleri infection and pericardial abscess associated with esophageal carcinoma: report of two cases. Hepatogastroenterology 1999;46:1782-1784.

30 Murarka S, Pranav F, Dandavate V: Pyogenic liver abscess secondary to disseminated streptococcus anginosus from sigmoid diverticulitis. J Glob Infect Dis 2011;3:79-81. 